\title{
A Non-Parametric Empirical Method for Nonlinear and Non-Stationary Signal Analysis
}

\author{
Yaakoub Berrouche \\ LIS laboratory, Department of Electronics \\ Faculty of Technology \\ Ferhat Abbes Sétif 1 University \\ Sétif, Algeria \\ berrouchey@gmail.com
}

\begin{abstract}
A Non-parametric Ensemble Empirical Mode Decomposition (NCEEMD) method is a novel technique for nonlinear and non-stationary signal analysis to detect a gearbox fault. The NCEEMD method was based on the CEEMD, but the Gaussian white noise was replaced by the fractional Gaussian noise. The NCEEMD method does not need to choose the appropriate SNR and the number of ensemble trials before signal processing, which makes it a non-parametric method. This new approach was evaluated using a simulated malfunction signal representing two typical faults in gearbox systems: modulation and rub-impact. Its performance was evaluated in terms of MSE and computation time. A comparative study between the EMD, EEMD, CEEMD, and NCEEMD methods showed that the latter performed better by improving the computation time and accuracy of CEEMD. The proposed method is a non-parametric method that provides a powerful tool in extracting the modulation and the rub-impact features from a vibration signal. The NCEEMD method helps to track down the gearbox faults and resolve this crucial problem in mechanical machines.
\end{abstract}

Keywords-complementary ensemble empirical mode decomposition; fractional Gaussian noise; gearbox fault detection; nonlinear signal analysis

\section{INTRODUCTION}

Gears are common and vital components in rotating machines, having a typical nonlinear and non-stationary signal. Monitoring the condition of a gear can help in the early detection of potential failures and avoidance of damages [1]. The predictions are based on the analysis of vibration signals generated by mechanical equipment. Many conventional methods of signal processing, such as statistical methods and Fourier analysis, have been applied to the analysis of vibration signals to diagnose gearbox faults. However, these methods are based on the assumption that the signals are stationary and linear, while gear defects have the nature of non-stationary processes [1].

Several methods have been proposed to deal with nonstationary signals. Among these techniques, the wavelet transform has good localization properties in the time and frequency domains, and it has been applied to the detection of damage in gear systems [2, 3]. However, the main drawback of the wavelet transform is the definitive choice of the wavelet base function before it is used [1,4], making it non-adaptive. The Empirical Mode Decomposition (EMD) was developed as a powerful tool to analyze nonlinear and non-stationary signals [5], and it has been applied in machine fault diagnosis [1,610]. EMD decomposes a signal into a finite number of Intrinsic Mode Functions (IMFs) by taking advantage of the signal's local properties. However, the main drawback of EMD is the mode mixing problem, where oscillations of different time scales can appear in one IMF, or oscillations with the same time scale can appear in different IMFs. To overcome the mode mixing problem, the Ensemble Empirical Mode Decomposition (EEMD) was proposed as an improved method of EMD [11]. EEMD is a noise-assisted data analysis method where the true IMFs are defined as the mean of an ensemble of trials, each consisting of the original signal plus a finite white noise. EEMD has been applied to the diagnosis of rotating machinery faults [12-14]. However, the resulting IMFs can be corrupted by additive white noise. The residue noise can be decreased by using a large number of ensemble trials $\left(N_{e}\right)$, resulting in high computation times. The Complementary EEMD method (CEEMD) was proposed to reduce computational times [15]. For this purpose, the white noise is added and subtracted separately from the original signal to generate two sets of IMFs. The average of IMFs of the same order could effectively reduce the final white noise residue.

This study proposes a new method named NCEEMD to enhance the performance of CEEMD by reducing the number of ensemble trials and therefore the amount of calculation and computation time. The NCEEMD method is based on the principles of the CEEMD, but the Gaussian white noise is replaced by fractional Gaussian noise $[16,17]$. The proposed method was evaluated using a simulated malfunction signal caused by modulation and rub-impact that represent two typical faults in gearbox systems. Its performance was evaluated in terms of fault diagnosis and computation time, and it was compared with the performance of EMD, EEMD, and CEEMD methods.

\section{MATHEMATICAL THEORY}

\section{A. EMD Algorithm}

The EMD method decomposes any non-linear and nonstationary signal into a finite number of IMF components by 
taking advantage of the local properties of the signal. This process is applied without requiring an a priori basis as the wavelet method by using a sifting algorithm. So, the original signal can be obtained by superposing the obtained $M-1$ IMFs and one residual $r(t)$ as [5]:

$$
s(t)=\sum_{j=1}^{M-1} I M F_{j}(t)+r(t)
$$

A more detailed description is provided in [5]. However, the main disadvantage of EMD is the problem of mode mixing, which affects the decomposition accuracy and the interpretation of the results.

\section{B. EEMD Algorithm}

The EEMD was proposed in [11] to overcome the mode mixing problem of EMD. The EEMD method defines the true IMF as the mean of an ensemble of trials, each consisting of the investigated signal corrupted by an additive white noise [11]. For a given number of ensemble trials $N_{e}$, a finite white noise is added to the signal in each trial, and then this noisy signal is decomposed into IMFs by using the EMD method [11]. Eventually, the true IMFs are obtained by averaging the IMFs of the same order. A complete description can be found in [11]. The EEMD method removes the problem of mode mixing. However, the resulting IMFs will be contaminated by the added noise. The added noise in the decomposed results can be reduced by using a large number of ensemble trials, increasing computation time.

\section{CEEMD Algorithm}

The CEEMD method was proposed to reduce the computational time of EEMD and the white noise residue. In this approach, white noise is added and subtracted separately from the original signal to generate two sets of IMFs with positive and negative noises [15]. The average of IMFs of the same order could eliminate the noise residue in the final IMFs. A drawback of CEEMD is the selection of the right level of added white noise and the number of adequate trials before each signal processing.

\section{NCEEMD Algorithm}

This study proposes the NCEEMD method to further reduce the number of ensemble trials. This method is based on the CEEMD method, but the Gaussian white noise was replaced by the Fractional Gaussian Noise (FGN). The FGN is a stationary process obtained by periodically sampling the fractional Brownian motion process $B_{H}(t)$ and by computing the first difference, defined by [17]:

$$
x(n)=B_{H}(n)-B_{H}(n-1)
$$

The autocorrelation of FGN is given by:

$$
R_{x}(k)=\frac{\sigma^{2}}{2}\left(|k-1|^{2 H}-2|k|^{2 H}+|k+1|^{2 H}\right)
$$

The FGN is characterized by the Hurst exponent $H$, where $0<H<1$, and its variance $\sigma^{2}$. For $H=1 / 2$ the FGN process is a white noise process, while other values produce nonzero correlations. For $0<H<1 / 2$ the correlation is negative, the autocorrelation decays very rapidly, and the frequency response looks like a high-pass filter. For $1 / 2<H<1$ the correlation is positive and the autocorrelation decays very slowly. The frequency response is similar to a low-pass filter.

\section{E. Hurst Exponent Selection}

The FGN is characterized by a Hurst exponent $H$ which is defined in two intervals. Two criteria were used in a systematic approach to select an appropriate $H$ and ensure a successful NCEEMD decomposition. The first criterion was to determine the values of $H$ that provide decomposition without mode mixing and no redundant IMF components. Figure 1(a) represents the different results of NCEEMD decomposition for the simulated malfunction signal described in Section III, according to the Hurst exponent where a value of 0 represents no redundant IMF component and the value of -1 represents the mode mixing [18]. Therefore, $H$ within the range of $[0,1 / 2]$ is a good value for a successful NCEEMD decomposition. Furthermore, it has been shown that the bandwidth of each IMF in the EMD method was much narrower for $H$ lower than $1 / 2$, and it increased with $H$ greater than $1 / 2$ [16]. Then, to ensure that the NCEEMD method behaves like an effective filter without mode mixing, the range $0<H<1 / 2$ must be chosen.

The second criterion was used to determine the exact value of $H$ in the selected range. To reach this aim, the accuracy of the decomposition using RMSE between the IMF1 and the rubimpact component of the simulated malfunction signal was evaluated according to the Hurst exponent, as shown in Figure 1(b). As it can be noted, the method has fewer errors in the range of $0.16<H<0.20$, while RMSE has a minimum at $H=0.185$. Therefore, the appropriate value of the Hurst exponent is $H=0.185$.

(a)

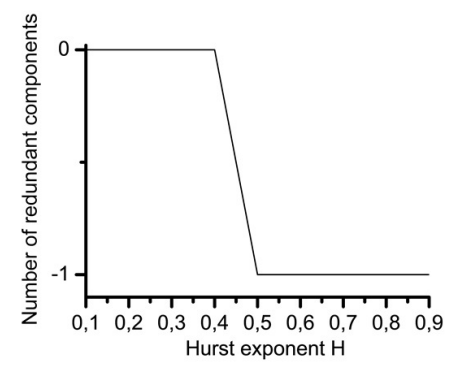

(b)

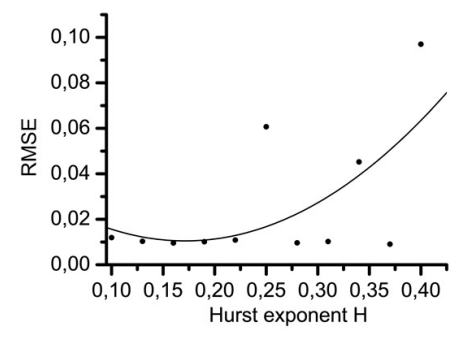

Fig. 1. Hurst exponent selection: (a) Relationship between the Hurst exponent and decomposition results obtained from NCEEMD, (b) Relationship between the Hurst exponent and RMSE (NCEEMD method).

\section{SIMULATION EXPERIMENTS}

Simulation experiments were conducted on a gearbox signal model, as an example of a non-linear and non-stationary 
signal, to analyze the performance of the proposed method and compare it with the conventional empirical method.

\section{A. Gearbox Signal Model}

The vibration signal, acquired from a normal gearbox, composed of a pair of gears and meshing under a constant load and speed, is represented by [19]:

$$
x(t)=\sum_{m=0}^{M} X_{m} \cos \left(2 \pi m N f_{s} t+\varphi_{m}\right)
$$

where $M$ is the number of tooth-meshing harmonics, $X_{m}$ and $\varphi_{m}$ are the amplitude and the phase modulation function of the $m^{\text {th }}$ meshing harmonic, respectively, $N$ is the teeth number of the gear, $f_{s}$ is the rotating frequency, and $N f_{s}$ is the meshing frequency. When gears with faults are meshing, the amplitude and the phase of the vibration signal would be modulated. The gear fault vibration signal can be expressed by [19]:

$$
y(t)=\sum_{m=0}^{M} X_{m}\left(1+a_{m}(t)\right) \cos \left(2 \pi m N f_{s} t+\varphi_{m}+b_{m}(t)\right)
$$

where $a_{m}(t)$ and $b_{m}(t)$ are periodic functions whose frequencies are the rotating frequency and its multiples that include the fault information of the gear vibration signal.

In this study, the normal gear vibration signal was used with one periodic function of the $m^{\text {th }}$ meshing harmonic of the amplitude and phase. When gears with a fault are meshing, both the amplitude and phase of this signal are modulated by the same periodic functions $\left(\left(a_{m}(t)=b_{m}(t)\right)\right.$. In addition to the modulation, the gear fault vibration signal can also include a rub-impact fault. Therefore, all the decomposition methods mentioned in Section II were tested and compared on a simulation signal that included modulation and rub-impact components.

\section{B. Application of NCEEMD on the Gearbox Signal}

The NCEEMD and two conventional methods were tested on a simulated gearbox signal as an example of a nonlinear and non-stationary signal. Two typical faults exist in a fault diagnosis of a gearbox: modulation and rub-impact. When the local rub-impact occurs in the gear, it produces an impact signal characterized by pulses of very short duration whose amplitude decreases exponentially. In addition, the normal gear vibration signal, with one periodic function of the $m^{\text {th }}$ meshing harmonic, was modulated by amplitude and phase modulation. The rub-impact signal, the amplitude, the phase-modulating signal, and the simulated malfunction signal that combines them are shown in Figure 2.

The simulated malfunction signal was decomposed by NCEEMD and the conventional methods EMD and EEMD to extract the rub-impact component and detect a fault, as shown in Figures 3-5. By comparing the decomposed components, NCEEMD successfully extracted the various components embedded in the simulated malfunction signal without mode mixing. This best performance in terms of RMSE was obtained with one trial and a low level of added FGN $(\mathrm{SNR}=25 \mathrm{~dB})$. On the other hand, EMD failed to extract the rub-impact component due to mode mixing between different IMFs. Likewise, EEMD extracted the rub-impact component but with a high computation time (number of ensembles $N_{e}=200$ ) and high error rate. These results suggest that NCEEMD is a sufficient method to diagnose a gearbox signal.
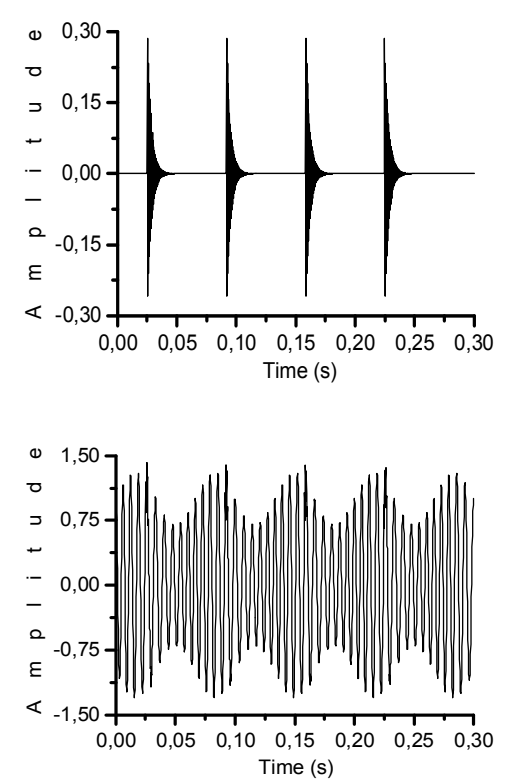

Fig. 2. Gearbox signal: (a) The rub-impact signal, (b) The simulated malfunction signal.

(a)

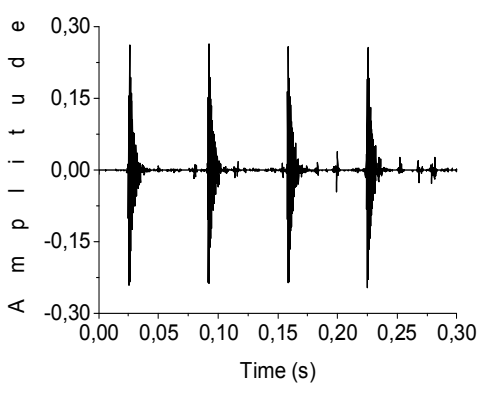

(b)

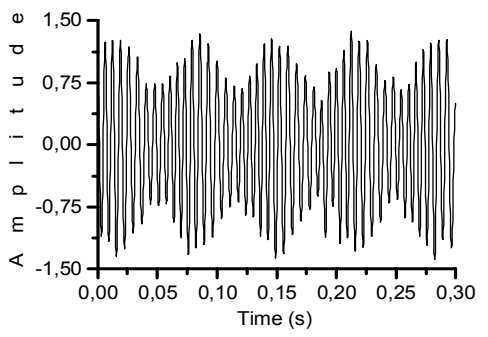

Fig. 3. The decomposed two components obtained by NCEEMD. (a) IMF1, (b) IMF2.

In conventional empirical methods, the level of noise added and the number of ensemble trials are the two primordial parameters needed to ensure a successful decomposition. Therefore, to measure the performance of NCEEMD and show that it can solve the problem of choosing the level of white noise added and determine the number of ensemble trials before processing, the appropriate number of ensemble trials is calculated by evaluating the correlation between the IMF1 and the high-frequency component in the simulated malfunction signal. 
(a)

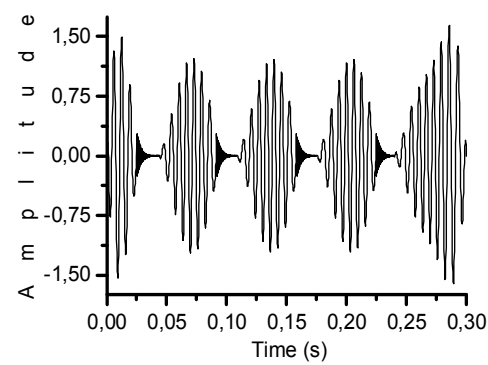

(b)

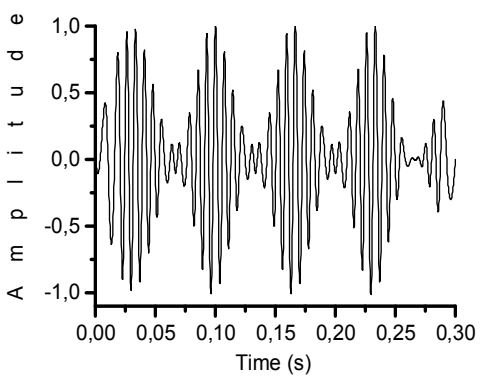

Fig. 4. The decomposed two components obtained by EMD. (a) IMF1, (b) IMF2.

(a)

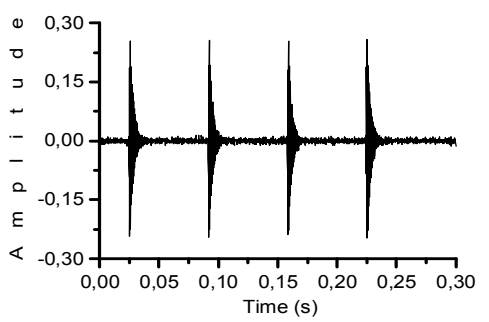

(b)

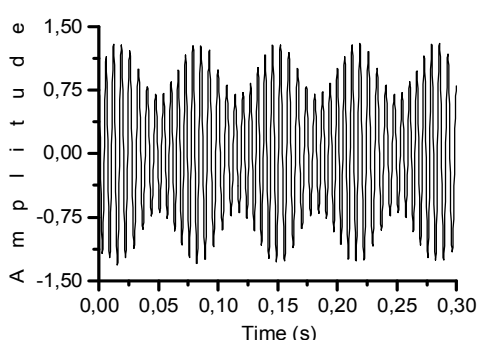

Fig. 5. The decomposed two components obtained by EEMD. (a) IMF1, (b) IMF2.

The relationship between the number of ensemble trials and the decomposition results obtained by EEMD (SNR=20dB), CEEMD (SNR=10dB), and NCEEMD are shown in Figure 6. These results show that NCEEMD requires a high SNR and one trial to reach a $95 \%$ of correlation. This means that the proposed method needs a low level of noise added with one trial to ensure successful decomposition. On the other hand, conventional methods need low SNR and a large number of ensemble trials before signal processing. Furthermore, the proposed method is non-parametric and decomposes any nonlinear and non-stationary signal in low computation time and high accuracy, compared to the classical methods.
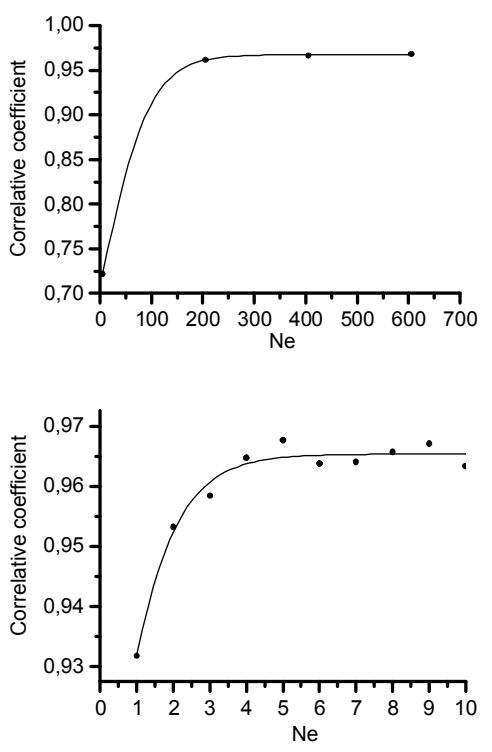

(c)

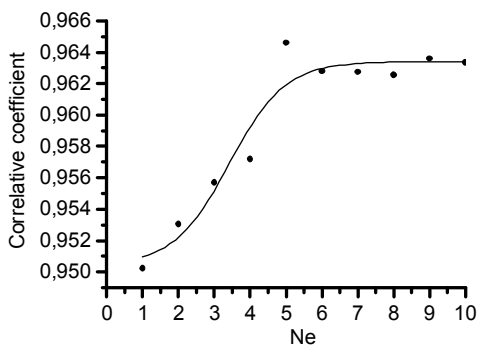

Fig. 6. Relationship between the correlation coefficient and the number of ensemble trials: (a) EEMD, (b) CEEMD, (c) NCEEMD.

\section{CONCLUSION}

Gearbox fault detection is a crucial task in mechanical machines. This paper proposed an efficient fault detection technique by introducing a new method named NCEEMD. Three techniques were compared, namely EEMD, CEEMD, and NCEEMD. Simulation experiments showed that EEMD, CEEMD, and NCEEMD managed to extract the gear meshing and the rub-impact fault components from a gearbox signal. However, the EEMD and CEEMD methods used a large number of ensemble trials and a high level of added noise that caused large computational time and more contamination with noise. However, the proposed NCEEMD method achieved better performance in terms of accuracy and computational time, without choosing any parameters before signal processing. Therefore, the proposed method is non-parametric and more adequate to deal with a nonlinear and non-stationary signal. 


\section{REFERENCES}

[1] S. J. Loutridis, "Damage detection in gear systems using empirical mode decomposition," Engineering Structures, vol. 26, no. 12, pp. 1833-1841, Oct. 2004, https://doi.org/10.1016/j.engstruct.2004.07.007.

[2] X. Wang, V. Makis, and M. Yang, "A wavelet approach to fault diagnosis of a gearbox under varying load conditions," Journal of Sound and Vibration, vol. 329, no. 9, pp. 1570-1585, Apr. 2010, https://doi.org/10.1016/j.jsv.2009.11.010.

[3] J. Rafiee, M. A. Rafiee, and P. W. Tse, "Application of mother wavelet functions for automatic gear and bearing fault diagnosis," Expert Systems with Applications, vol. 37, no. 6, pp. 4568-4579, Jun. 2010, https://doi.org/10.1016/j.eswa.2009.12.051.

[4] Z. K. Peng and F. L. Chu, "Application of the wavelet transform in machine condition monitoring and fault diagnostics: a review with bibliography," Mechanical Systems and Signal Processing, vol. 18, no. 2, pp. 199-221, Mar. 2004, https://doi.org/10.1016/S0888-3270 (03)00075-X.

[5] N. E. Huang et al., "The empirical mode decomposition and the Hilbert spectrum for nonlinear and non-stationary time series analysis," Proceedings of the Royal Society of London. Series A: Mathematical, Physical and Engineering Sciences, vol. 454, no. 1971, pp. 903-995, Mar. 1998, https://doi.org/10.1098/rspa.1998.0193.

[6] G. Gai, "The processing of rotor startup signals based on empirical mode decomposition," Mechanical Systems and Signal Processing, vol. 20, no. 1, pp. 222-235, Jan. 2006, https://doi.org/10.1016/j.ymssp.2004.07.001.

[7] C. Junsheng, Y. Dejie, and Y. Yu, "The application of energy operator demodulation approach based on EMD in machinery fault diagnosis," Mechanical Systems and Signal Processing, vol. 21, no. 2, pp. 668-677, Feb. 2007, https://doi.org/10.1016/j.ymssp.2005.10.005.

[8] J. Cheng, D. Yu, J. Tang, and Y. Yang, "Application of frequency family separation method based upon EMD and local Hilbert energy spectrum method to gear fault diagnosis," Mechanism and Machine Theory, vol. 43, no. 6, pp. 712-723, Jun. 2008, https://doi.org/10.1016/ j.mechmachtheory.2007.05.007.

[9] Q. Gao, C. Duan, H. Fan, and Q. Meng, "Rotating machine fault diagnosis using empirical mode decomposition," Mechanical Systems and Signal Processing, vol. 22, no. 5, pp. 1072-1081, Jul. 2008, https://doi.org/10.1016/j.ymssp.2007.10.003.

[10] F. Wu and L. Qu, "Diagnosis of subharmonic faults of large rotating machinery based on EMD," Mechanical Systems and Signal Processing, vol. 23, no. 2, pp. 467-475, Feb. 2009, https://doi.org/10.1016/ j.ymssp.2008.03.007.

[11] Z. Wu and N. E. Huang, "Ensemble empirical mode decomposition: a noise-assisted data analysis method," Advances in Adaptive Data Analysis, vol. 1, no. 1, pp. 1-41, Jan. 2009, https://doi.org/10.1142/ S1793536909000047.

[12] Y. Lei, Z. He, and Y. Zi, "Application of the EEMD method to rotor fault diagnosis of rotating machinery," Mechanical Systems and Signal Processing, vol. 23, no. 4, pp. 1327-1338, May 2009, https://doi.org/ 10.1016/j.ymssp.2008.11.005.

[13] Y. Lei, Z. He, and Y. Zi, "EEMD method and WNN for fault diagnosis of locomotive roller bearings," Expert Systems with Applications, vol. 38, no. 6, pp. 7334-7341, Jun. 2011, https://doi.org/10.1016/ j.eswa.2010.12.095.

[14] Y. Zhou, T. Tao, X. Mei, G. Jiang, and N. Sun, "Feed-axis gearbox condition monitoring using built-in position sensors and EEMD method," Robotics and Computer-Integrated Manufacturing, vol. 27, no. 4, pp. 785-793, Aug. 2011, https://doi.org/10.1016/j.rcim.2010.12.001.

[15] J. R. Yeh, J. S. Shieh, and N. E. Huang, "Complementary ensemble empirical mode decomposition: a novel noise enhanced data analysis method," Advances in Adaptive Data Analysis, vol. 2, no. 2, pp. 135156, Apr. 2010, https://doi.org/10.1142/S1793536910000422.

[16] G. Rilling, P. Flandrin, and P. Goncalves, "Empirical mode decomposition, fractional Gaussian noise and Hurst exponent estimation," in Proceedings. (ICASSP '05). IEEE International Conference on Acoustics, Speech, and Signal Processing, 2005., Mar. 2005, vol. 4, pp. 489-492 Vol. 4, https://doi.org/10.1109/ICASSP. 2005.1416052 .
[17] V. Ingle, S. Kogon, and D. Manolakis, Statisical and Adaptive Signal Processing. Northwood, MA, US: Artech, 2005.

[18] J. Zhang, R. Yan, R. X. Gao, and Z. Feng, "Performance enhancement of ensemble empirical mode decomposition," Mechanical Systems and Signal Processing, vol. 24, no. 7, pp. 2104-2123, Oct. 2010, https://doi.org/10.1016/j.ymssp.2010.03.003.

[19] X. Fan and M. J. Zuo, "Gearbox fault detection using Hilbert and wavelet packet transform," Mechanical Systems and Signal Processing, vol. 20, no. 4, pp. 966-982, May 2006, https://doi.org/10.1016/ j.ymssp.2005.08.032.

[20] R. E. Bekka and Y. Berrouche, "Improvement of ensemble empirical mode decomposition by over-sampling," Advances in Adaptive Data Analysis, vol. 5, no. 3, Jul. 2013, Art. no. 1350012, https://doi.org/ 10.1142/S179353691350012X.

[21] I. Tellala, N. Amardjia, and A. Kesmia, "A Modified EMD-ACWA Denoising Scheme using a Noise-only Model," Engineering, Technology \& Applied Science Research, vol. 10, no. 2, pp. 5470-5476, Apr. 2020, https://doi.org/10.48084/etasr.3406.

[22] W. Mohguen and S. Bouguezel, "Denoising the ECG Signal Using Ensemble Empirical Mode Decomposition," Engineering, Technology \& Applied Science Research, vol. 11, no. 5, pp. 7536-7541, Oct. 2021, https://doi.org/10.48084/etasr.4302.

[23] A. Y. Al-Rawashdeh, "Investigation of an Induction Wound Rotor Motor to Work as a Synchronous Generator," Engineering, Technology \& Applied Science Research, vol. 9, no. 2, pp. 4071-4074, Apr. 2019, https://doi.org/10.48084/etasr.2606. 